\title{
Tenodermodesis para el tratamiento del dedo en martillo tendinoso de presentación tardía en niños y adolescentes
}

\author{
Andrés Ferreyra, Pablo L. Eamara, Lucas M. Lanfranchi, Victoria M. Allende Nores, Julio J. Masquijo \\ Departamento de Ortopedia y Traumatología Infantil, Sanatorio Allende, Córdoba, Argentina
}

\begin{abstract}
RESUMEN
Introducción: El diagnóstico del dedo en martillo tendinoso puede pasar desapercibido inicialmente en niños y adolescentes, esto limita las posibilidades del tratamiento conservador. El objetivo fue evaluar los resultados del tratamiento quirúrgico con la técnica de tenodermodesis en lesiones de presentación tardía. Materiales y Métodos: Se evaluó retrospectivamente a 9 pacientes (8 niños) con una edad promedio de $8.6 \pm 6$ años (rango 1-15). Los días promedio de evolución de la lesión eran $27 \pm 11.4$ (rango 15-45). El mecanismo de lesión fue una herida cortante (4 casos) y un traumatismo indirecto (5 casos). El tratamiento consistió en tenodermodesis e inmovilización transitoria con clavija transarticular. El seguimiento promedio fue de $61 \pm 34.7$ meses (rango 12-106). Se evaluaron la movilidad activa y pasiva de la articulación interfalángica distal, la presencia de dolor o deformidad, la limitación de actividades de la vida diaria y la necesidad de tratamientos adicionales. Se clasificaron los resultados con los criterios de evaluación de Crawford. Resultados: En 8 pacientes, el resultado fue excelente y, en uno, regular según Crawford. Un paciente poco colaborador requirió una segunda intervención por re-rotura. En dos casos, hubo una complicación (granuloma) y requirió resección. Ningún paciente refirió dolor al final del seguimiento, ni limitaciones para las actividades de la vida diaria. Ocho presentaron extensión activa completa y uno, una deformidad residual de $20^{\circ}$. Conclusión: La tenodermodesis permite la reconstrucción anatómica del mecanismo extensor en niños y adolescentes. Los resultados clínicos de este estudio son alentadores en lesiones no diagnosticadas en forma temprana.
\end{abstract}

Palabras clave: Dedo en martillo; tenodermodesis; presentación tardía; pediátrico.

Nivel de Evidencia: IV

Tenodermodesis for the Treatment of Late-Presenting Soft Tissue Mallet Finger Deformities in Children and Adolescents

\begin{abstract}
Introduction: Diagnosis of soft tissue mallet finger may go unnoticed initially in children and adolescents, limiting the possibilities of conservative treatment. The aim of this study was to evaluate the results of surgical treatment with the tenodermodesis technique in late-presentig lesions. Materials and Methods: Nine patients (8 male) with an average age 8.6 \pm 6 years (range 1-15 years) were retrospectively evaluated. The lesion presented at an average of $27 \pm 11.4$ days after trauma (range 15 to 45 days). In 4 patients the mechanism was a laceration and in 5 indirect trauma. Patients were treated by tenodermodesis and transitory fixation of the distal interphalangeal joint with a Kirshner-wire. The average follow-up was $61 \pm 34.7$ months (range 12-106 months). Active and passive mobility of the distal interphalangeal joint (DIPJ), pain, deformity, limitation of activities of daily life, and need for further treatment were evaluated. Crawford criteria was used to evaluate the outcomes. Results: The results were excellent in eight patients, and fair in one according to the Crawford criteria. One case required reoperation for re-rupture in a poorly collaborating patient. Two cases presented as a complication a granuloma and required resection of it. No patient presented pain at the last follow-up, nor limitations for activities of daily living. Eight patients had full active DIPJ extension, and one had a $20^{\circ}$ residual deformity. Conclusion: Tenodermodesis allows anatomical reconstruction of the extensor mechanism in pediatric patients. The clinical results are encouraging in late-presenting lesions.
\end{abstract}

Key words: Mallet finger; tenodermodesis; late presentation; pediatric.

Level of Evidence: IV

Recibido el 21-7-2019. Aceptado luego de la evaluación el 27-6-2020 • Dr. ANDRÉS FERREYRA • and_ferreyra@yahoo.com.ar ID https://orcid.org/0000-0003-1163-7114 Cómo citar este artículo: Ferreyra A, Eamara PL, Lanfranchi LM, Allende Nores VM, Masquijo JJ. Tenodermodesis para el tratamiento del dedo en martillo tendinoso de presentación tardía en niños y adolescentes. Rev Asoc Argent Ortop Traumatol 2021;86(1):23-30. https://doi.org/10.15417/issn. 1852-7434.2021.86.1.1006 


\section{INTRODUCCIÓN}

Las roturas del aparato extensor a nivel de la articulación interfalángica distal (IFD) pueden ocasionar una lesión de dedo en martillo, ${ }^{1-6}$ que pueden ser tendinosa u ósea. Típicamente provocan un déficit de extensión e impotencia funcional para extender activamente la articulación IFD. ${ }^{4} \mathrm{El}$ mecanismo de lesión suele ser un traumatismo en la punta del dedo o incluso lesiones abiertas. ${ }^{78}$ En niños y adolescentes, el diagnóstico precoz puede pasar desapercibido, tanto por el edema inicial como por la dificultad que implica examinar a un niño pequeño. ${ }^{7}$ Incluso el déficit de extensión no suele ser tan evidente en el primer examen. ${ }^{7}$ Cuando se diagnostica y trata apropiadamente, en algunos pacientes, la inmovilización no es mantenida en forma adecuada, por lo que, en algunos casos, se desarrolla una lesión crónica. ${ }^{7,9}$ La mayoría de los autores clasifican a las lesiones como agudas cuando son tratadas antes de las dos semanas ${ }^{10-12} \mathrm{y}$ crónicas luego de las cuatro semanas. ${ }^{1,10,13}$ Altan y cols. consideran como lesiones tardías a aquellas que son tratadas luego de dos semanas. ${ }^{11}$

Se han descrito múltiples técnicas para tratar el dedo en martillo tendinoso crónico: tenotomía de la bandeleta central, reconstrucción del ligamento retinacular oblicuo espiral, reinserción con arpones, artrodesis, condrodesis, entre otras..$^{1,-6,13-15}$ En 1977, Iselin y cols. ${ }^{9}$ comunicaron la tenodermodesis. Esta técnica tiene la ventaja de realizar un aumento al tendón extensor con tejido celular subcutáneo y piel de espesor completo, lo que mejoraría la integridad mecánica y la vascularización de la zona, e incrementaría el potencial de cicatrización. ${ }^{4}$ Además, técnicamente es más sencilla que las otras opciones mencionadas. ${ }^{4,6,7,9}$

Se han publicado muchos resultados de la técnica de tenodermodesis en la población adulta. ${ }^{1,2,6,9,13,16-19}$ Sin embargo, se dispone de escasa información en pacientes pediátricos. ${ }^{4,7}$ El objetivo de este estudio fue evaluar los resultados obtenidos con la técnica de tenodermodesis en una serie de niños y adolescentes con lesiones de dedo en martillo tendinoso de presentación tardía.

\section{MATERIALES Y MÉTODOS}

Luego de la aprobación por el Comité de Ética de la institución, se revisaron retrospectivamente las historias clínicas de los pacientes pediátricos con diagnóstico de dedo en martillo tendinoso, que se sometieron a cirugía con la técnica de tenodermodesis, con más de 15 días de evolución desde la lesión, entre enero de 2008 y abril de 2016. Todos fueron operados en la misma institución, por dos ortopedistas especializados en niños. Para el análisis se incluyó a nueve pacientes ( 8 niños y 1 niña), con una edad promedio de $8.6 \pm 6$ años (rango 1-15). El tiempo promedio de evolución de la lesión era de $27 \pm 11.4$ días (rango 15-45). El mecanismo de la lesión fue una herida cortante ( 4 casos) y un traumatismo indirecto del dedo ( 5 casos). El seguimiento mínimo fue de 12 meses (rango 12-106) (Tabla 1).

Tabla 1. Datos demográficos de los pacientes

\begin{tabular}{|c|c|c|c|c|c|c|c|c|}
\hline n & Edad & Sexo & Mano & Dedo & Mecanismo & $\begin{array}{c}\text { Tiempo de } \\
\text { evolución (días) }\end{array}$ & $\begin{array}{l}\text { Tratamiento } \\
\text { previo }\end{array}$ & $\begin{array}{l}\text { Seguimiento } \\
\text { (meses) }\end{array}$ \\
\hline 1 & 15 & M & $\mathrm{D}$ & $5^{\text {to }}$ & $\begin{array}{c}\text { Trauma indirecto } \\
\text { (rugby) }\end{array}$ & 30 & No & 106 \\
\hline 2 & 4 & M & $\mathrm{D}$ & $3^{\text {ro }}$ & Corte (vidrio) & 15 & Sutura de piel & 91 \\
\hline 3 & 2 & M & I & $3^{\text {ro }}$ & Corte (bicicleta) & 21 & Sutura de piel & 89 \\
\hline 4 & 15 & $\mathrm{~F}$ & I & $4^{\text {to }}$ & $\begin{array}{l}\text { Trauma indirecto } \\
\text { (voleibol) }\end{array}$ & 20 & No & 80 \\
\hline 5 & 9 & M & $\mathrm{D}$ & $4^{\text {to }}$ & $\begin{array}{l}\text { Trauma indirecto } \\
\text { (pelotero) }\end{array}$ & 30 & No & 75 \\
\hline 6 & 4 & M & $\mathrm{D}$ & $3^{\text {ro }}$ & $\begin{array}{l}\text { Aplastamiento } \\
\text { (puerta) }\end{array}$ & 45 & $\begin{array}{c}\text { Reducción y } \\
\text { osteosíntesis con } \\
\text { clavija } 2^{\text {da }} \text { falange }\end{array}$ & 55 \\
\hline 7 & 13 & M & I & $4^{\text {to }}$ & Corte (hacha) & 30 & Sutura de piel & 34 \\
\hline 8 & 15 & M & $\mathrm{D}$ & $5^{\text {to }}$ & Trauma indirecto & 15 & No & 14 \\
\hline 9 & 1 & M & $\mathrm{D}$ & $2^{\text {do }}$ & Corte & 45 & Sutura de piel & 12 \\
\hline
\end{tabular}




\section{Técnica quirúrgica y manejo posoperatorio}

Se utilizó la misma técnica quirúrgica en todos los pacientes. El procedimiento se realizó en quirófano bajo sedación con internación ambulatoria. Se administró una dosis profiláctica de cefazolina por vía intravenosa, según el peso, 30 minutos antes de la incisión. Se colocó al paciente en decúbito supino, con el miembro sobre una mesa de mano y manguito hemostático. Se fijó transitoriamente la articulación IFD con una clavija retrógrada (Figura 1). Se realizó un abordaje dorsal sobre el pliegue de la articulación IFD, que puede ser ampliado en forma de S o de H (Figura 2), según la preferencia del cirujano. Se identificaron ambos extremos del tendón extensor, se los disecó cuidadosamente y se comprobó que pudieran ser aproximados sin tensión. Se suturó en un solo plano el tendón, el tejido celular subcutáneo y la piel con material no reabsorbible monofilamento (Figura 3). Se colocaron un vendaje estéril y una férula metálica digital. La clavija transarticular fue retirada en el consultorio a las 4-7 semanas, según el criterio del cirujano.
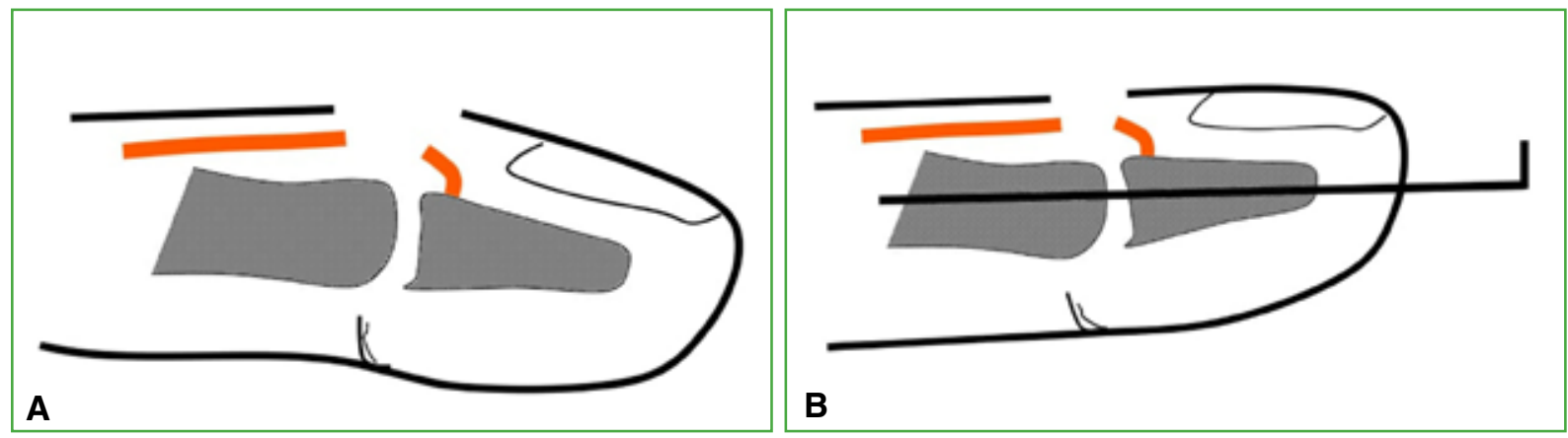

Figura 1. A. Defecto de extensión inicial. B. Fijación interfalángica transitoria con clavija retrógrada.

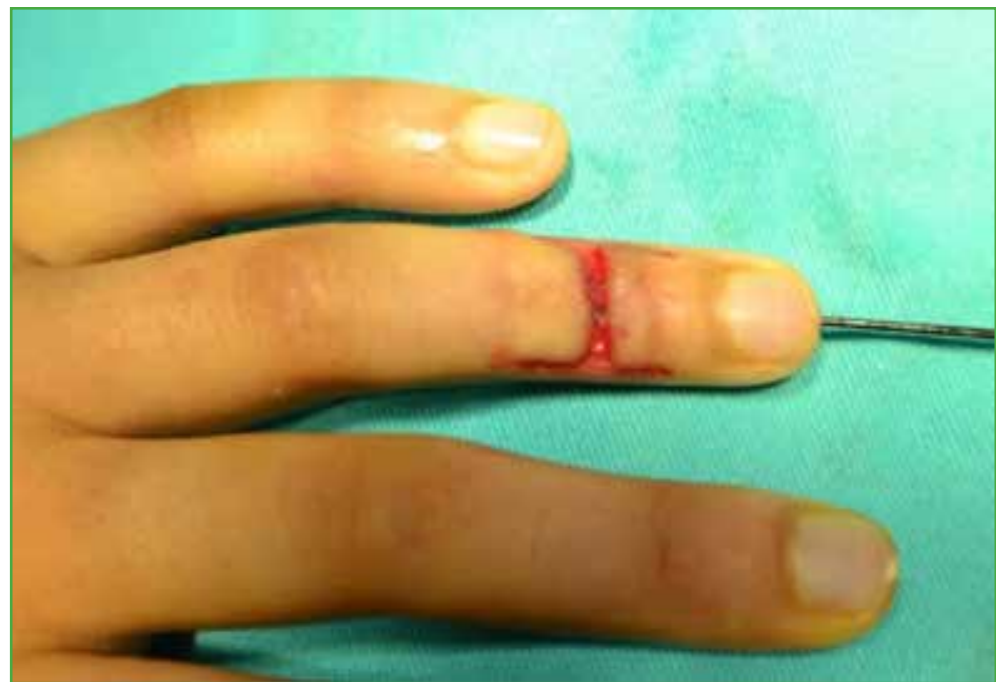

Figura 2. Abordaje en forma de $\mathrm{H}$ para el tratamiento quirúrgico del dedo en martillo con tenodermodesis. La rama transversal debe estar a nivel del pliegue dorsal de la articulación interfalángica distal. 

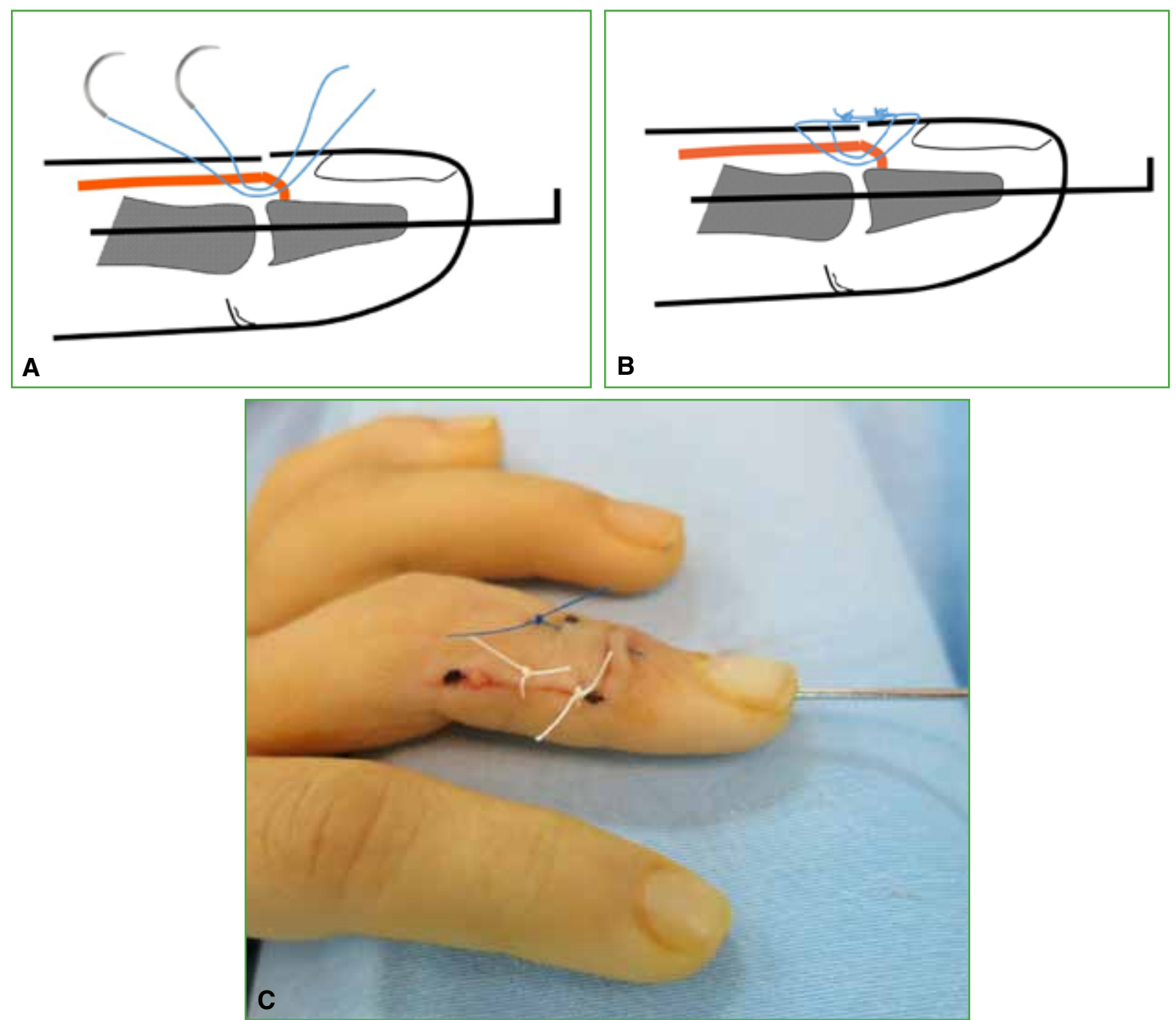

Figura 3. A y B. Sutura con material no reabsorbible monofilamento, que incluye el tendón, el tejido celular subcutáneo y la piel. C. Imagen clínica de la herida suturada en un paciente con abordaje en forma de $\mathrm{S}$.

\section{Evaluación de los pacientes}

Al final del seguimiento, se evaluó la movilidad activa y pasiva de la articulación IFD, la presencia de dolor o deformidad, la limitación de actividades de la vida diaria y la necesidad de tratamientos adicionales. Se utilizaron los criterios de evaluación de Crawford ${ }^{20}$ para clasificar los resultados, se considera que un resultado es excelente si la extensión de la articulación IFD y la flexión son completas, y no hay dolor; bueno si hay un déficit de extensión de $0-10^{\circ}$, flexión completa y no hay dolor; regular cuando el déficit de extensión es hasta de $25^{\circ}$, cualquier grado de pérdida de flexión y no hay dolor; y pobre cuando el déficit de extensión es $>25^{\circ}$ o hay dolor persistente (Tabla 2). 
Tabla 2. Criterios de Crawford ${ }^{20}$

\begin{tabular}{|l|l|}
\hline Grado & Descripción \\
\hline Excelente & $\begin{array}{l}\text { Extensión completa de la articulación interfalángica distal, } \\
\text { flexión completa, sin dolor }\end{array}$ \\
\hline Bueno & Déficit de extensión de $0^{\circ}-10^{\circ}$, flexión completa, sin dolor \\
\hline Regular & $\begin{array}{l}\text { Déficit de extensión de } 0^{\circ}-25^{\circ}, \text { cualquier pérdida de flexión, } \\
\text { sin dolor }\end{array}$ \\
\hline Pobre & Déficit de extensión $>25^{\circ}$ o dolor persistente \\
\hline
\end{tabular}

\section{Análisis estadístico}

Para la descripción de las variables cuantitativas se utilizó una estadística descriptiva (promedio, desviación estándar y rango) y, para las variables cualitativas, se utilizaron las frecuencias absolutas.

\section{RESULTADOS}

Se obtuvieron excelentes resultados en ocho pacientes y un resultado regular en el restante, según los criterios de Crawford $^{20}$ (Tabla 3). Todos los pacientes realizaban sus actividades de la vida diaria sin restricciones ni dolor al final del seguimiento. La extensión de la articulación IFD fue completa en todos los casos (Figura 4), excepto uno con una deformidad residual de $20^{\circ}$ (caso 6).

Tabla 3. Tratamiento, resultados, complicaciones y seguimiento

\begin{tabular}{|c|c|c|c|c|}
\hline $\mathbf{n}$ & $\begin{array}{c}\text { Tiempo de inmovilización } \\
\text { (semanas) }\end{array}$ & Resultado* & Complicación & No \\
\hline 1 & 7 & Excelente & No & Resección de granuloma \\
\hline 2 & 5 & Excelente & Granuloma & Resección de granuloma \\
\hline 3 & 5 & Excelente & Granuloma & No \\
\hline 4 & 6 & Excelente & No & No \\
\hline 5 & 6 & Excelente & No & Revisión \\
\hline 6 & 6 & Regular & Re-rotura & No \\
\hline 7 & 5 & Excelente & No & No \\
\hline 8 & 7 & Excelente & No & No \\
\hline 9
\end{tabular}




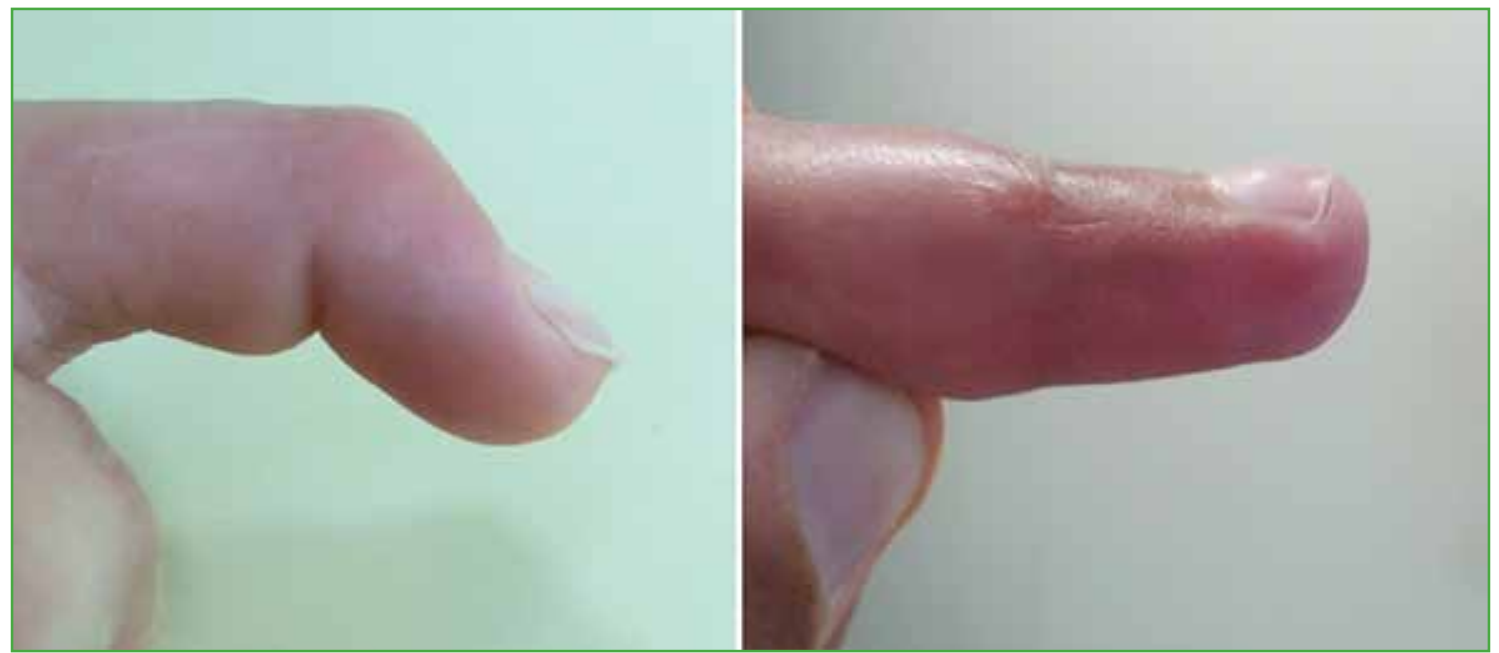

Figura 4. Aspecto clínico antes del tratamiento y una vez corregida la deformidad.

Los dedos más afectados fueron el tercero y el cuarto ( 3 casos cada uno), el quinto ( 2 casos) y el segundo ( 1 caso). Cinco pacientes fueron tratados tardíamente debido a un diagnóstico erróneo en la etapa aguda. Estos fueron los cuatro casos con herida cortante, a los que solo se les había realizado una sutura de la piel y un paciente con una fractura de la falange media del mismo dedo, que fue tratado con reducción y osteosíntesis con clavija percutánea. En este caso, el diagnóstico se realizó al retirar la clavija y constatar el déficit de extensión de la articulación IFD. Los restantes cuatro pacientes consultaron tardíamente.

Tres requirieron nuevas intervenciones: un caso de pérdida de la fijación transitoria a las dos semanas de la cirugía y re-rotura de la reparación. Se revisó la tenodermodesis y se obtuvo un buen resultado; otros dos pacientes presentaron un granuloma en la herida que requirió resección.

\section{DISCUSIÓN}

Las lesiones de dedo en martillo son frecuentes en niños y adolescentes. ${ }^{4}$ La rotura del aparato extensor en la articulación IFD puede ser secundaria a una avulsión de la epífisis o, con menos frecuencia, a una lesión tendinosa. Cuando se diagnostican precozmente, el tratamiento conservador suele ser eficaz en la mayoría de los casos. ${ }^{1,3,10,11,19,21,22}$ Sin embargo, hay lesiones que pueden ser diagnosticadas de forma tardía, porque pasan desapercibidas o la inmovilización no se realizó o mantuvo de manera correcta. Los pacientes que consultan tarde suelen tener dolor y dificultades para las actividades de la vida diaria, y están disconformes con el aspecto estético. ${ }^{18}$

El tratamiento ideal del dedo en martillo tendinoso de presentación tardía o crónico es controvertido. ${ }^{1,3,6}$ Algunos autores recomiendan intentar un tratamiento conservador con inmovilización durante 6-8 semanas antes de indicar la cirugía. ${ }^{4}$ Se han comunicado buenos resultados con este enfoque en adultos. ${ }^{2,10,11,22}$ No existen series que evalúen el tratamiento conservador en pacientes pediátricos con dedo en martillo crónico. Sin embargo, debido al potencial de cicatrización de los niños, sería lógico pensar que los resultados serían aun mejores que en la población adulta. Consideramos que es conveniente intentar un tratamiento conservador, al menos, durante 6-8 semanas antes de indicar la cirugía.

Se han descrito numerosas técnicas quirúrgicas. La tenotomía de la bandeleta central descrita por Fowler permite balancear el aparato extensor al resecar la inserción de la bandeleta central, lo que incrementa la excursión y la fuerza de la inserción distal, pero requiere incidir un sector previamente sano del tendón. En una serie de pacientes adultos, uno de cada cuatro casos tuvo deformidad residual con esta técnica. ${ }^{23}$ La reconstrucción del ligamento retinacular oblicuo requiere el uso de un injerto libre del tendón palmar mayor. ${ }^{24,25} \mathrm{La}$ reinserción con arpones busca convertir una lesión crónica en una aguda, suturando el tendón a la falange distal, ${ }^{14}$ aunque requeriría cruentar la inserción distal en forma extensa, con riesgo de lesionar la fisis. La artrodesis tiene la desventaja de limitar la movilidad de una articulación sana. ${ }^{26}$ La tenodermodesis es un procedimiento técnicamente sencillo comparado 
con otras opciones. ${ }^{4,6,79} \mathrm{Al}$ reparar la lesión tendinosa junto con la piel y el tejido celular subcutáneo, permite una reconstrucción mecánicamente más resistente y provee un aporte vascular adicional para la cicatrización. ${ }^{4}$ Se han publicado numerosas series de pacientes adultos. Iselin y cols. comunicaron 22 resultados satisfactorios en 26 pacientes. ${ }^{9}$ En la serie de Warren y cols., cuatro de seis pacientes mejoraron, mientras que uno no tuvo cambios y el restante empeoró. ${ }^{16}$ Kon y Bloem publicaron 26 resultados excelentes en 27 pacientes. ${ }^{17}$ Sorene y Goodwin afirman que la tenodermodesis permitió mejorar el déficit de extensión de $50^{\circ}$ a $9^{\circ}$, en una serie de 16 pacientes, aunque con cierta limitación de la flexión. ${ }^{6}$ Solo se han publicado dos artículos sobre la tenodermodesis para tratar lesiones de dedo en martillo tendinoso crónicas en la población pediátrica (Tabla 4). ${ }^{4,7}$ De Boeck y Jaeken ${ }^{7}$ comunicaron cuatro casos con excelentes resultados. Kardestuncer y cols. ${ }^{4}$ obtuvieron ocho resultados excelentes y dos buenos o regulares en su serie de 10 pacientes. Los resultados de nuestra serie son comparables con los publicados por estos autores.

Tabla 4. Comparación de publicaciones sobre tenodermodesis para dedo en martillo crónico en niños

\begin{tabular}{|c|c|c|c|c|c|c|}
\hline Autores & n & Edad* & $\begin{array}{c}\text { Tiempo } \\
\text { lesión-cirugía* }\end{array}$ & $\begin{array}{l}\text { Tiempo de } \\
\text { inmovilización* } \\
\text { (semanas) }\end{array}$ & $\begin{array}{c}\text { Resultados } \\
\text { (Criterios } \\
\text { de Crawford) }\end{array}$ & $\begin{array}{l}\text { Seguimiento* } \\
\text { (meses) }\end{array}$ \\
\hline $\begin{array}{l}\text { De Boeck y } \\
\text { Jaeken }\end{array}$ & 4 & $\begin{array}{c}8.5 \\
(7-12)\end{array}$ & $\begin{array}{l}6.5 \text { meses } \\
(4-9)\end{array}$ & $\begin{array}{l}4.75 \\
(3-6)\end{array}$ & 4 Excelente & $42(8-72)$ \\
\hline \multirow[t]{2}{*}{ Kardestuncer y cols. ${ }^{4}$} & \multirow[t]{2}{*}{10} & \multirow{2}{*}{$\begin{array}{c}7.4 \\
(1.4-17.8)\end{array}$} & \multirow[t]{2}{*}{$\mathrm{NE}$} & \multirow{2}{*}{$\begin{array}{l}\mathrm{NE} \\
(4-6)\end{array}$} & 8 Excelente & \multirow[t]{2}{*}{$78(12-152)$} \\
\hline & & & & & $\begin{array}{l}2 \text { Bueno - } \\
\text { Regular }\end{array}$ & \\
\hline \multirow[t]{2}{*}{ Estudio actual } & \multirow[t]{2}{*}{9} & \multirow[t]{2}{*}{$\begin{array}{c}8.6 \\
(1-15)\end{array}$} & \multirow[t]{2}{*}{$\begin{array}{l}27.8 \text { días } \\
(15-45)\end{array}$} & \multirow[t]{2}{*}{$\begin{array}{c}5.6 \\
(4-7)\end{array}$} & 8 Excelente & \multirow[t]{2}{*}{$61(12-106)$} \\
\hline & & & & & 1 Regular & \\
\hline
\end{tabular}

*En promedio (rango). $\mathrm{NE}=$ No especificado.

Este estudio tiene limitaciones propias del diseño metodológico que deben ser mencionadas. La muestra analizada es retrospectiva y relativamente pequeña, aunque similar a las otras dos series pediátricas tratadas con la misma técnica. ${ }^{4,7}$ El tiempo de evolución de la lesión fue registrado de acuerdo con la anamnesis a los padres, pero podría presentar algunas inconsistencias, ya que algunos no recordaban exactamente el momento del trauma. A pesar de estas limitaciones, creemos que este estudio brinda más información sobre los resultados de la cirugía en pacientes pediátricos con lesiones de presentación tardía.

En nuestra serie, la tenodermodesis permitió la reconstrucción anatómica del mecanismo extensor en niños y adolescentes con lesiones de dedo en martillo tendinoso y mejorar la extensión del dedo afectado sin limitar la flexión. Los resultados clínicos de este estudio son alentadores en lesiones no diagnosticadas en forma temprana.

Conflicto de intereses: Los autores no declaran conflictos de intereses.

ORCID de P. Eamara: https://orcid.org/0000-0001-7739-3568 ORCID de L. M. Lanfranchi: https://orcid.org/0000-0002-5009-5293
ORCID de V. M. Allende Nores: https://orcid.org/0000-0003-4893-7276 ORCID de J. J. Masquiijo: https://orcid.org/0000-0001-9018-0612 


\section{BIBLIOGRAFÍA}

1. Alla SR, Deal ND, Dempsey IJ. Current concepts: mallet finger. Hand 2014;9(2):138-44. https://doi.org/10.1007/s11552-014-9609-y

2. Bendre AA, Hartigan BJ, Kalainov DM. Mallet finger. J Am Acad Orthop Surg 2005;13(5):336-44. https://doi.org/10.5435/00124635-200509000-00007

3. Valdes K, Naughton N, Algar L. Conservative treatment of mallet finger - A systematic review. J Hand Ther 2015;28(3):237-45. https://doi.org/10.1016/j.jht.2015.03.001

4. Kardestuncer T, Bae DS, Waters PM. The results of tenodermodesis for severe chronic mallet finger deformity in children. J Pediatr Orthop 2008;28(1):81-5. https://doi.org/10.1097/BPO.0b0131815ff31e

5. Shin EK, Bae DS. Tenodermodesis for chronic mallet finger deformities in children. Tech Hand Up Extrem Surg 2007;11(4):262-5. https://doi.org/10.1097/BTH.0b013e31812f5714

6. Sorene ED, Goodwin DR. Tenodermodesis for established mallet finger deformity. Scand J Plast Reconstr Surg Hand Surg 2004;38(1):43-5. https://doi.org/10.1080/02844310310009528

7. De Boeck H, Jaeken R. Treatment of chronic mallet finger deformity in children by tenodermodesis. J Pediatr Orthop 1992;12(3):351-4. https://doi.org/10.1097/01241398-199205000-00013

8. Waters PM, Benson LS. Dislocation of the distal phalanx epiphysis in toddlers. J Hand Surg Am 1993;18(4):581-5. https://doi.org/10.1016/0363-5023(93)90293-C

9. Iselin F, Levame J, Godoy J. A simplified technique for treating mallet fingers: tenodermodesis. J Hand Surg Am 1977;2(2):118-21. https://doi.org/10.1016/s0363-5023(77)80095-6

10. Garberman SF, Diao E, Peimer CA. Mallet finger: Results of early versus delayed closed treatment. J Hand Surg Am 1994;19:850-2. https://doi.org/10.1016/0363-5023(94)90200-3

11. Altan E, Alp NB, Baser R, Yalçın L. Soft-tissue mallet injuries: a comparison of early and delayed treatment. $J$ Hand Surg Am 2014;39(10):1982-5. https://doi.org/10.1016/j.jhsa.2014.06.140

12. Auchincloss JM. Mallet-finger injuries: a prospective, controlled trial of internal and external splintage. Hand 1982;14(2):168-73. https://doi.org/10.1016/s0072-968x(82)80011-9

13. Bellemere P. Treatment of chronic extensor tendons lesions of the fingers. Chir Main 2015;34(4):155-81. https://doi.org/10.1016/j.main.2015.05.001

14. Makhlouf VM, Deek NA. Surgical treatment of chronic mallet finger. Ann Plast Surg 2011;66(6):670-2. https://doi.org/10.1097/SAP.0b013e3181e6d017

15. Ulkür E, Cengiz A, Ozge E, Celiköz B. Repair of chronic mallet finger deformity using Miteck micro arc bone anchor. Ann Plast Surg 2005;5:393-6. https://doi.org/10.1097/01.sap.0000151464.03967.a2

16. Warren RA, Kay NR, Ferguson DG. Mallet finger: comparison between operative and conservative management in those cases failing to be cured by splintage. J Hand Surg Br 1988;13(2):159-60. https://doi.org/10.1016/0266-7681(88)90127-1

17. Kon M, Bloem JJAM. Treatment of mallet fingers by tenodermodesis. Hand 1982;14(2):174-5. https://doi.org/10.1016/s0072-968x(82)80012-0

18. Nakamura K, Nanjyo B. Reassessment of surgery for mallet finger. Plast Reconstr Surg 1994;93:141-149. PMID: 8278469

19. Doyle JR. Extensor tendons-acute injuries. En: Green DP, Hotchkiss RN, Pederson WC (eds). Green's operative hand surgery, $4^{\text {th }}$ ed. New York, NY: Churchill Livingstone; 1999:1962-87.

20. Crawford FP. The molded polythene splint for mallet finger deformities. Hand Am 1984;2(9):231-7. https://doi.org/10.1016/s0363-5023(84)80148-3

21. Georgescu AV, Capota IMV, Matei IRG. A new surgical treatment for mallet finger deformity- Deepithelialised pedicled skin flap technique. Injury 2013;44:351-5. https://doi.org/10.1016/j.injury.2013.01.013

22. Brzezienski MA, Schneider LH. Extensor tendon injuries at the distal interphalangeal joint. Hand Clin 1995;11:37386. PMID: 7559816

23. Houpt P, Dijkstra R, Storm van Leeuwen JB. Fowler's tenotomy for mallet deformity. J Hand Surg Br 1993;18:499500. https://doi.org/10.1016/0266-7681(93)90157-b

24. Thompson JS, Littler JW, Upton J. The spiral oblique retinacular ligament (SORL). J Hand Surg Am 1978;3:482-7. https://doi.org/10.1016/s0363-5023(78)80144-0

25. Kleinman WB, Petersen DP. Oblique retinacular ligament reconstruction for chronic mallet finger deformity. $J$ Hand Surg Am 1984;9:399-404. https://doi.org/10.1016/s0363-5023(84)80231-2

26. Katzman SS, Gibeault JD, Dickson K, Thompson JD. Use of a Herbert screw for interphalangeal joint arthrodesis. Clin Orthop 1993;296:127-32. PMID: 8222414 